\title{
Regeneration of the pancreatic $\beta$ cell
}

\author{
Massimo Trucco \\ Division of Immunogenetics, Department of Pediatrics, University of Pittsburgh School of Medicine, \\ Children's Hospital of Pittsburgh, Pittsburgh, Pennsylvania, USA.
}

\begin{abstract}
Type 1 diabetes is the result of an autoimmune attack against the insulin-producing $\beta$ cells of the endocrine pancreas. Current treatment for patients with type 1 diabetes typically involves a rigorous and invasive regimen of testing blood glucose levels many times a day along with subcutaneous injections of recombinant DNA-derived insulin. Islet transplantation, even with its substantially improved outcome in recent years, is still not indicated for pediatric patients. However, in light of the fact that some regenerative capabilities of the endocrine pancreas have been documented and recent research has shown that human ES cell lines can be derived in vitro, this review discusses whether it is practical or even possible to combine these lines of research to more effectively treat young diabetic patients.
\end{abstract}

In vertebrates, the process of gastrulation takes place very early during the development of the embryo. This process reorganizes the embryo's cells into 3 layers: ectoderm, endoderm, and mesoderm. The ectoderm forms the skin and the central nervous system; the mesoderm gives rise to the cells from which blood, bone, and muscle are derived; and the endoderm forms the respiratory and digestive tracts $(1,2)$. The embryonic endoderm, taking the shape of the primitive gut tube, serves as a template for the gastrointestinal tract from which the embryonic pancreas eventually buds. It has been shown that the branching morphogenesis of the pancreatic bud gives rise to the ducts and the acinar components of the gland. Endocrine progenitors, proliferating from the budding ducts, then form aggregates of differentiated cells known as the islets of Langerhans (Figure 1). While the pancreatic acini, composed of cells dedicated to the secretion into the intestine of enzymes that will participate in the digestive process of the ingested food, constitute the exocrine component of the gland, the islets are made up of 4 cell populations, organized in a stereotypical topological order, which constitute instead the endocrine component of the gland. In the islet, the $\alpha$ cells produce glucagon; the $\beta$ cells, insulin; the $\gamma$ cells, pancreatic polypeptide; and the $\delta$ cells, somatostatin $(1,2)$ (Figure 1 ).

It is in large part due to this knowledge of pancreatic embryogenesis that residual pancreatic endocrine progenitors, able to guarantee islet homeostasis, were originally postulated to be still present in the pancreatic ducts of the adult gland. The identification and characterization of these putative progenitors is of paramount importance, not only for a better understanding of endocrine pancreatic physiology and pathology, but also for the development of potential therapeutic approaches that their correct exploitation may offer.

Type 1 diabetes is the clinical consequence of the destruction of the insulin-producing $\beta$ cells of the pancreas, mediated by autoreactive $T$ cells specifically directed against $\beta$ cell determinants (3). The loss of the majority of the $\beta$ cell population, evident at the onset of the disease, requires daily subcutaneous injections of quantities of insulin that should be proportional to the quantity of glucose present in the blood at each moment in time. The physical replacement of the $\beta$ cell mass constitutes the rationale for which islet transplan-

Nonstandard abbreviations used: ALS, anti-lymphocyte serum; BMP4, bone morphogenetic protein 4; GIP, glucose-dependent insulinotropic polypeptide; MDC, muscle-derived cell; PMP, pancreas-derived multipotent precursor.

Conflict of interest: The author has declared that no conflict of interest exists.

Citation for this article: $J$. Clin. Invest. 115:5-12 (2005).

doi:10.1172/JCI200423935. tation was originally proposed by Paul Lacy (4). Although it was recently demonstrated that islet cell transplants can be performed with greater chances of success than just a few years ago (5), the constrains under which this is clinically possible are still too numerous to allow the broad application of this procedure to permanently cure the disease. The immunosuppressive drug regimen necessary to protect islets from a recurrent autoimmune response and allorejection may, with time, irreversibly damage kidney function, while the process of islet isolation itself, even if drastically improved during the last few years, damages transplantable islets and, consequently, two to three donors are necessary in order to obtain the minimal cell mass sufficient for transplantation into a single recipient (6).

In light of recent discoveries showing the regenerative capabilities of the endocrine pancreas and the in vitro derivation of human ES cell lines, we have to consider possible, non-mutually exclusive alternatives to allogeneic islet transplantation.

\section{Does the elusive pancreatic stem cell exist?}

There exists, in humans, a stem cell committed to a specific lineage that is capable of giving rise to all types of differentiated cells and tissues, including extraembryonic tissues: the totipotent cell. The mammalian zygote perhaps should be considered the preeminent totipotent stem cell by antonomasia. However, in utero, this stem cell continues to divide and becomes an amalgam of similar, but not identical, daughter cells. We do not yet know how to distinguish among these daughter cells the few that continue to have the capacity to regenerate the whole, multivariate, final product - if these totipotent cells still exist at all. Consequently, we do not yet have specific markers capable of characterizing the totipotent cell. Once the various tissues and organs begin to form, we do not know whether any totipotent cells are preserved within them and, if they are, how long they could continue to be functional. Intuitively, we can argue that precursors of some kind are present and active within our body throughout life, because even elderly people are able to repair damaged tissues, albeit with reduced efficiency. However, we still do not know where these hypothetical precursor cells may be hiding and which final differentiated cells they can in fact generate.

Teleologically, our regenerative system should have developed according to the same rationale that underlies the stationing of firehouses throughout an entire city to allow each unit to be able to more rapidly reach the fire location and efficiently intervene. That is, it should have deployed into each organ not necessarily totipotent cells, but at least precursors with self-maintenance capabilities, as well as those necessary to replace worn-out 

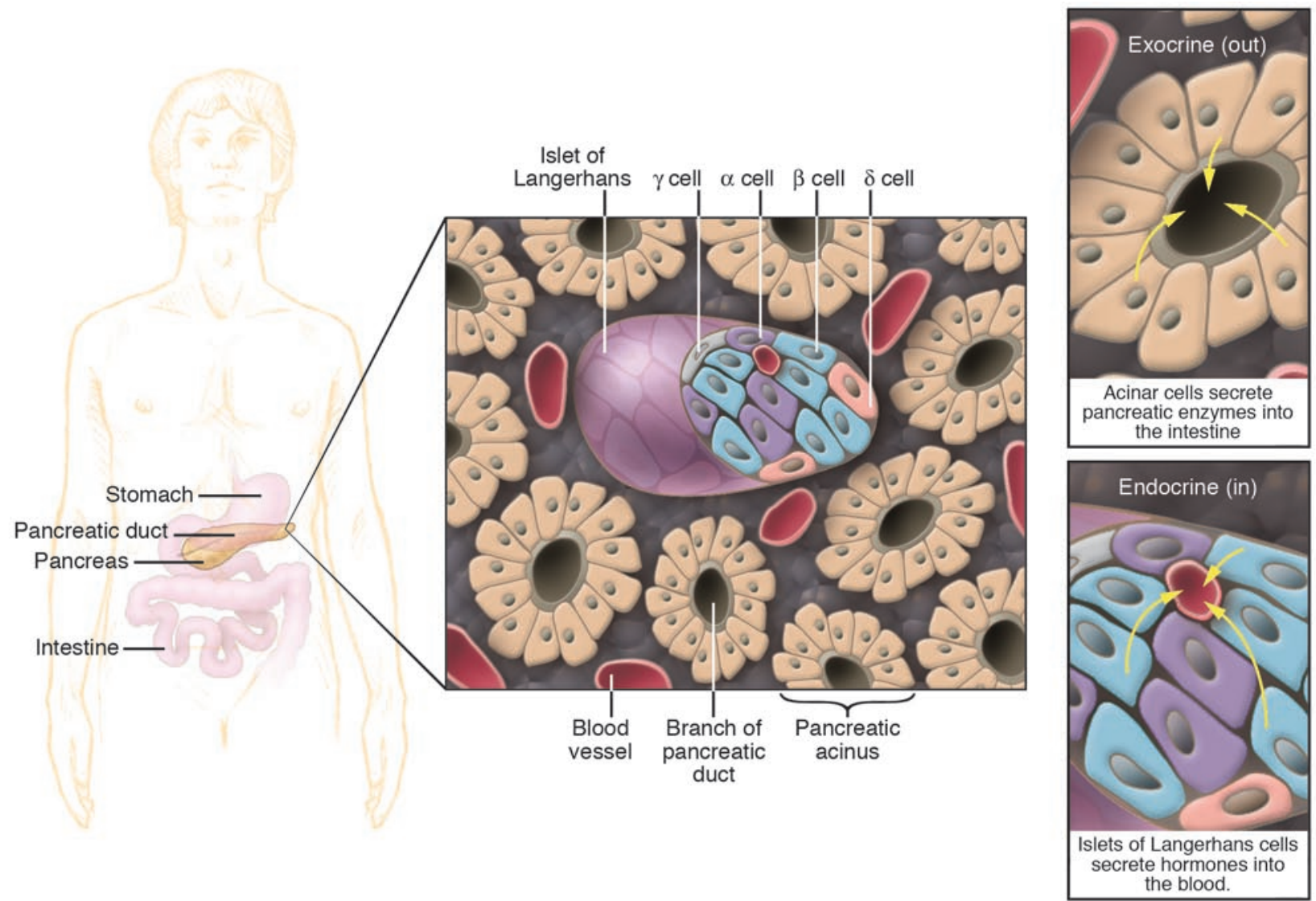

Figure 1

Cross section of the pancreas. The pancreas houses 2 distinctly different tissues. Its bulk comprises exocrine tissue, which is made up of acinar cells that secrete pancreatic enzymes delivered to the intestine to facilitate the digestion of food. Scattered throughout the exocrine tissue are many thousands of clusters of endocrine cells known as islets of Langerhans. Within the islet, $\alpha$ cells produce glucagon; $\beta$ cells, insulin; $\delta$ cells, somatostatin; and $\gamma$ cells, pancreatic polypeptide - all of which are delivered to the blood.

cells. These precursors should be able to support a process that, while progressing relatively slowly in the maintenance of tissue homeostasis, could be converted, in case of crisis, into a rapidly operating system. Consequently, this system would be highly effective at responding promptly to a number of stimulations, promoted by nervous signaling, microarchitectural remnants of destroyed tissues, metabolites of energy generating processes, peptide growth factors, etc. (7-9). Physiologically, in the endocrine pancreas, hormone-producing cells that are at the end of their life span should be continuously, albeit quite slowly, replaced by newly generated cells. We need to learn more about this regenerative process if we wish to take advantage of it for therapeutic purposes.

In considering the utility of stem cells for the regeneration of the $\beta$ pancreatic cell, we currently face some major questions: in the adult endocrine pancreas, do multipotent progenitors still exist? If so, where exactly are they located? What are the best markers for recognizing and isolating them? What are the stimuli able to activate their differentiation pathway(s)? How large is the time window in which they can still respond to the needs of an aging tissue? In the absence of pancreatic progenitors, are there adult pluripotent stem cells - cells not committed to a specific lineage that may differentiate into all types of cells and tissues, with the exception of extraembryonic tissues - located elsewhere in the body that may have the capability to regenerate $\beta$ cell mass? If these cells are all lineage-committed precursor cells, can we instead use ES cell lines derived in vitro to substitute the lost $\beta$ cells of the pancreas?

\section{The road to regeneration}

Increases in $\beta$ cell mass may occur through increased $\beta$ cell replication, increased $\beta$ cell size, decreased $\beta$ cell death, and differentiation of possibly existing $\beta$ cell progenitors (10). It has been shown that occasional endocrine cells can be found embedded in normal pancreatic ducts. However, these cells are few and far between (11). The number of these duct-associated endocrine cells physiologically increases as the consequence of severe insulin resistance in obese individuals or during pregnancy $(12,13)$. Similar histological changes are observed under conditions of tissue injury and repair after partial pancreatectomy, duct ligation, cellophane wrapping of the gland, or IFN- $\gamma$ overexpression driven by the insulin promoter (14-17). Even then, within the ducts, only a small number of cells become insulin positive. This suggests that even if some hypothetical precursors exist, the process of formation of endocrine cells out of the islet (neogenesis) would not be a frequently observed property of the duct epithelium. On the other hand, the fact that $\alpha$ and $\beta$ cells develop from a possibly common, non-hormone-expressing, yet $P d x 1$-positive precursor $(\mathrm{Pdx} 1$ being a transcription factor required for pancre- 
atic development) suggests that all cell types found within the islet may originate from a bona fide, common endocrine progenitor (18). These endocrine progenitors may be located close to the duct but may not actually be components of the ductal epithelium (19). The progenitor cells could be mesenchymal in origin, or they could be cells differentiated from an unknown cell type. If the number of these progenitors is extremely small, lineage analysis becomes very difficult because of the lack of known appropriate markers. Moreover, if these cells are as rare as they appear to be, it becomes difficult to quantify their contribution to normal endocrine cell turnover.

These are some of the conclusions discussed by Weir and Bonner-Weir (20) in commenting on the study by Seaberg et al., in which it was shown that single murine adult pancreatic precursor cells can generate progeny with characteristics of pancreatic cells, including $\beta$ cells (21). These rare ( 1 in 3,000-9,000 cells) pancreasderived multipotent precursors (PMPs) do not seem to be bona fide pluripotent ES cells, since they lack, for example, the Oct4 and Nanog markers that direct the propagation of undifferentiated ES cells; nor are these cells of clear ectodermal, mesodermal, or endodermal origin, since they failed to express other markers considered specific for precursors of each of the embryonic cell types $(20,21)$. Because, surprisingly, these PMPs also lacked some $\beta$ cell markers (e.g., HNF3 $\beta$ ) as well as ductal epithelium markers (e.g., cytokeratin), but were able to generate differentiation products with neural characteristics along with $\alpha, \beta, \delta$, and acinar pancreatic cells, the authors proposed the existence of a new and unique ectodermal/endodermal precursor cell present during embryonic development that could persist in adult tissues (21).

These results support the conclusions of another recent study in which multipotent pancreatic progenitors were prospectively isolated using flow-cytometric cell sorting (22). The marker used in this case was c-Met, the HGF receptor. The rationale for this choice was the known signal exchange between epithelial and mesenchymal cells, promoting the interaction between c-Met and HGF, which plays an important role in the development of the pancreas. The authors suggest that c-Met-HGF interaction is critically responsible for growth and differentiation of pancreatic stem and progenitor cells not only during development but also in the adult, where they maintain homeostasis and promote regeneration. Colonies derived from single c-Met-positive cells, sorted from neonatal and adult mouse pancreatic tissues, contained cells expressing several markers for endocrine, acinar, and ductal lineage cells. While neuroectodermal markers were not evaluated, the isolated pancreatic stem cells in the study by Suzuki et al. (22) were also able to generate offspring cells expressing hepatocyte and gastrointestinal cell markers, possibly due to the selection marker used. Seaberg's PMP-derived cells were grown instead in the serum-free medium conditions normally used for neural stem cell culture (21).

All of these studies, even with their somewhat divergent outcomes, seem to support the conclusion that endocrine precursor cells of some kind exist in the pancreas. They are present not only in the duct, but also within the islets themselves, since both subpopulations were independently used as the source of the isolated single cell precursors (21). On the one hand, this conclusion supports the working hypothesis of those who propose that pancreatic ductal cells can transdifferentiate into $\beta$ cells and that this is a physiologic process generally more efficiently activated by increased metabolic demand and tissue injuries (23); on the other hand, it may also accommodate the most recent results of Dor and colleagues (24), who propose instead that no $\beta$ cell can arise from non $-\beta$ cell progenitors, whether in the normal adult pancreas or after pancreatectomy. As a direct consequence, the number of $\beta$ cells should become virtually defined at a certain point, and, afterward, glycemia should be controlled only by that defined cellular pool. Dor's results were obtained by using a sophisticated Cre/lox system that, in transgenic mice, can be induced by tamoxifen. This system labels fully differentiated $\beta$ cells (defined as postnatal cells transcribing the insulin gene) that express the human alkaline phosphatase protein, which is in turn revealed by a histochemical stain. In a defined period of time, the "chase," only the cells that are progeny of preexisting and labeled $\beta$ cells are newly labeled. New $\beta$ cells derived from any non- $\beta$ cell source, including stem cells, are not labeled. The frequency and distribution of labeled $\beta$ cells within pancreatic islets, at the end of the chase period, should be inversely proportional to the number of new, nonlabeled cells present in the same structures. If the frequency of labeled $\beta$ cells does not change, as was observed, the number of cells derived from the differentiation of non-insulin-producing precursors must be minimal or null, while terminally differentiated insulin-producing $\beta$ cells themselves should be the cells that actually proliferate and give rise to other insulin-producing $\beta$ cells. While the results of Seaberg et al. (21) do not contest the proven yet limited ability of a $\beta$ cell to divide, the failure of Dor et al. (24) to observe cells possibly differentiated from stem or precursor cells might actually be due to both their extremely limited number (21) and technical issues. For example, the use of tamoxifen injected intraperitoneally or subcutaneously twice a week for two and a half weeks (24) may have blocked neogenesis from precursor cells mainly in pancreatic ducts adjacent to involuted islets, as observed by Pelengaris et al. (25), and once the tamoxifen was withdrawn, these cells may not have had sufficient time to differentiate.

\section{Controlling the autoimmune response}

On this basis, we can tentatively conclude that precursors of a perhaps unconventional type can be located both in close proximity to and inside the endocrine tissue and that they can be activated by increased metabolic demand or by still-unknown secreted factors, normally able to accelerate the process that guarantees homeostasis of islets of Langerhans under normal conditions. The physiologic equilibrium between lost and newly generated cells can be altered by the action of $\beta$ cell-specific, autoreactive $\mathrm{T}$ cells in instances in which autoimmunity develops (3). Once $\mathrm{T}$ cell killing activity overcomes the regenerative compensatory activity of the organ, the number of functional $\beta$ cells progressively decreases until they become too few to maintain the glucohomeostasis of the entire body. The time of transition over this metabolic threshold becomes immediately evident with the presentation of the characteristic signs of the clinical onset of type 1 diabetes. During the course of disease, even if the regenerative properties of the pancreas remain functional, the continued presence of diabetogenic, autoreactive $T$ cells consistently nullifies the reparative effort. The fact that these autoreactive $T$ cells remain present in the body of the diabetic patient for a long time is proven by experiments in which healthy islet cells transplanted into syngeneic, long-term diabetic mice or humans were quickly killed by these same autoreactive T cells (26).

The autoimmune response is successfully averted in the NOD mouse either by directly eliminating the majority of the autoreactive $\mathrm{T}$ cells with anti-T cell antibodies or by substituting all or part of the immunocompetent cell repertoire with bone marrow cells obtained from diabetic-resistant donors. 

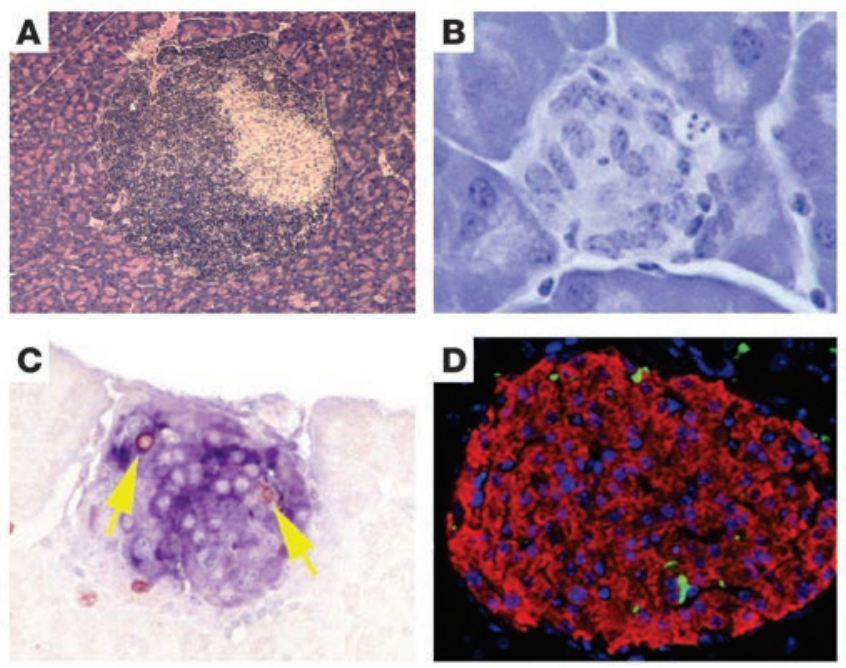

Figure 2

Regeneration of the $\beta$ cell in diabetic NOD mice. (A) In NOD mice, the infiltration of autoreactive T cells into the islets of Langerhans (resulting in insulitis) begins at around 4 weeks of age. At 20 to 23 weeks, approximately $85 \%$ of female mice are diabetic, i.e., their glycemia is greater than $300 \mathrm{mg} / \mathrm{dl}$. Magnification, $\times 200$. (B) When it is successfully transplanted with bone marrow from a non-diabetes-prone donor and hematopoietic chimerism is established, the NOD mouse no longer show signs of autoimmune activity. However, while there is no more evidence of insulitis in the endogenous pancreas, there is also no sign of insulin production (no red staining). Magnification, $\times 400$. (C) Insulinpositive cells in the islets can be seen to be dividing (yellow arrows); i.e., they are insulin (blue) and BrdU (red) positive. Magnification, $\times 400$. (D) Three to 4 months after bone marrow transplantation, new insulin-positive cells (shown in red) are present throughout the endogenous pancreas. Magnification, $\times 200$. Thus, when the islets transplanted under the kidney capsule in order to maintain euglycemia while regeneration takes place are removed by nephrectomy, the mice remain nondiabetic. Figure reproduced with permission from Stem Cells (33).

In a study by Ogawa et al., the treatment of overtly diabetic NOD mice with anti-lymphocyte serum (ALS) abrogated autoimmunity but achieved only partial clinical remission (27). Transient treatment of overtly diabetic NOD mice with ALS and exendin-4, a potent insulinotropic hormone that promotes replication and differentiation of $\beta$ cells in vitro and in vivo, achieved instead complete remission of $88 \%$ of the treated animals within 75 days, accompanied by progressive normalization of glucose tolerance, improved islet histology, increased insulin content in the pancreas, and almost normal insulin release in response to a glucose challenge. These results show that exendin-4 synergistically augments the remission-inducing effect of ALS, possibly by promoting differentiation of $\beta$ cell precursors (27).

\section{Figure 3}

Using a GFP-transgenic mouse as donor, it is possible to observe how the majority of the transplanted bone marrow cells do not directly participate in the regeneration of the endogenous pancreas. As shown here, there are no double-positive (orange) cells in the newly formed islets. The donor cells (green) appear to be located close to possibly existing juxta-ductal precursor cells, which may be activated by bone marrow cell-secreted factors. Insulin-positive cells are red. Magnification, $\times 400$. See also refs. 32 and 33.
Also, the successful induction of a mixed allogeneic chimerism obtained after transplanting bone marrow from a diabetes-resistant donor into a diabetic animal following a sublethal dose of irradiation is sufficient to block and eventually also revert the systematic invasion and inflammation of the islets by the autoreactive $\mathrm{T}$ cells that result in insulitis (Figure 2) (28-30). Within the endocrine pancreas, once the insult of autoimmunity is abrogated, the physiologic process of regeneration can continue efficiently, eventually replenishing the population of insulin-producing cells to a number sufficient to maintain euglycemia, thus curing the diabetic recipient (Figure 2D) (31-33). While this process takes place - and it is still debatable whether this occurs over weeks (32) or months (33) - the recipient's glycemia must be controlled by additional, independent measures. The most commonly used technique is to transplant into the recipient islets from the same marrow donor. However, the successful engraftment of the transplanted bone marrow, or the establishment of a steady hematopoietic chimerism, would have to be maintained without the use of immunosuppressive agents. These potent drugs may kill not only the still-present autoreactive T cells of the recipient, but also the $\beta$ cells themselves, thereby defeating the purpose of the transplant (34-36). The use of immunosuppressive agents may also interfere with the observed rise of regulatory $\mathrm{T}$ cells, a possible explanation for the long-lasting immunoregulatory cell-dominant condition observed in cured animals. Adoptive transfer experiments in which both diabetogenic lymphocytes and splenocytes from ALS-treated, long-term diabetes-free NOD mice were transplanted in NOD/SCID mice with no signs of diabetes induction support this hypothesis (27).

A subject of ongoing debate is whether either or both the transplanted bone marrow and the cotransplanted $\beta$ cells are necessary for promoting an efficient regenerative process, independent of their ability to block autoimmunity or preserve euglycemia, respectively. They may, for example, secrete factors such as glucagon-like peptides, which are useful in order to sustain an efficient regenerative process $(27,37,38)$. Strong evidence suggests that the hematopoietic precursors present in the bone marrow cell population do not directly participate in the reparative process of the insulin-producing cell population (Figure 3) $(32,33)$. In the cured recipient, insulin-producing cells that are genetically marked (by GFP or the Y chromosome of the male donor in the female recipient) to indicate they are of donor origin are extremely rare, occurring in no more than 2 of more than $100,000 \beta$ cells. These cells may actually be the result of sporadic cell fusion processes (39). A different source of donor cells, for example, the spleen, might be able to block autoimmunity and also provide mesenchymal $\beta$ cell precursors (40). However, the hypothesized presence in the mouse spleen of embryonic mesenchymal cells that

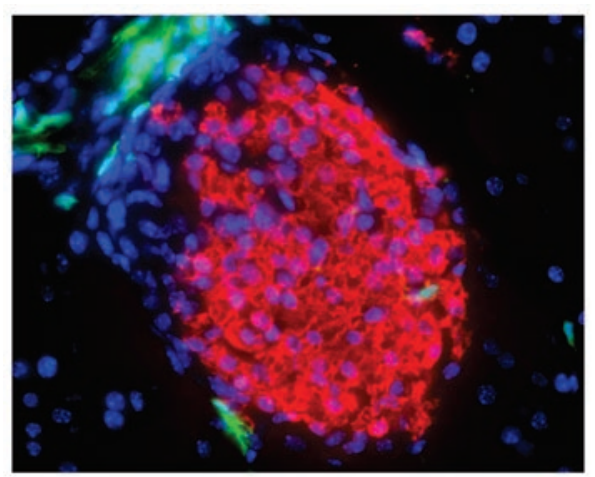




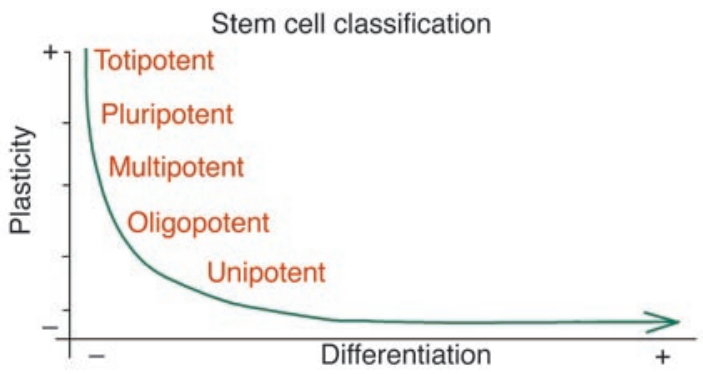

\section{Figure 4}

Classification of stem cells based on their developmental potential according to Wagers and Weissman (44). Totipotent, able to give rise to all embryonic and extraembryonic cell types; pluripotent, able to give rise to all cell types of the embryo proper; multipotent, able to give rise to a subset of cell lineages; oligopotent, able to give rise to a restricted subset of cell lineages; unipotent, able to contribute only one mature cell type.

lack surface expression of $\mathrm{CD} 45$ and are able to differentiate into endothelial and endodermal cells remains to be confirmed.

\section{Further questions}

Even when regeneration of the $\beta$ cells from precursor cells is definitively proven, issues still to be resolved will include the time frame and physiological conditions necessary for regeneration to occur and reach completion, as well as the circumstances that facilitate or limit the regeneration process and the ability of clinicians to promote or avoid these, to more efficiently achieve the desired therapeutic results. Also, assuming the existence of $\beta$ cell precursors, we still do not know whether these cells are immortal or subject to senescence, a situation that would leave a narrow window of time for intervention. This matter may be especially relevant in diabetic individuals in whom the reparative process has been repressed by autoimmune surveillance for a long period of time. If successful immunoregulatory intervention cannot be initiated immediately after the clinical onset of the disease, a full recovery of the endocrine function of the gland via the physiologic regeneration route may become impossible. However, if the regenerative process is proven to be irreversibly compromised at a certain point, it may still be possible to transplant into diabetic patients functional precursor cells from nondiabetic donors or to artificially convert the patient's own cells from other tissues or lineages into insulin-producing $\beta$ cells.

\section{The potential for ES cells}

Even if specific markers necessary to recognize $\beta$ cell precursors were available, the physical isolation of these cells from a patient's pancreas would not be an easy task. Increasing the number of possible precursors ex vivo while avoiding the activation of differentiation pathways would also be problematic, as would be eventually facilitating their differentiation toward the final product, in this case, a functional $\beta$ cell. Culturing and expanding $\beta$ cells in vitro has also been shown to be difficult and perhaps limited to a few proliferative cycles, since they are terminally differentiated cells. It has been postulated that it would be easier to derive precursor cells from the embryo and use them to regenerate the damaged endocrine pancreas. Although human ES cell lines have been successfully derived $(41,42)$ and recently made available to the scientific community (43), the need to direct their differentiation toward a specific final product, in this case the $\beta$ cell, still remains a major hurdle that must be overcome.
A stem cell is, by definition, the one cell capable of duplicating itself and resuming its undifferentiated status, while also originating progeny that can differentiate into one or more final products that are physiologically defined by their specific functions (44). Proceeding through the differentiation pathway, stem cells can be categorized as totipotent, pluripotent, multipotent, oligopotent, and unipotent, depending upon all their possibly reversible, progressively acquired characteristics (Figure 4) (44).

ES cells are pluripotent cell lines derived from the inner cell mass of blastocyst-stage embryos $(41-43,45)$, and their differentiation in culture may reproduce characteristics of early embryonic development. Based on similarities between mechanisms that control the development of both the adult pancreas and the central nervous system, Lumelsky et al. (46) hypothesized that strategies able to induce production of neural cells from ES cells could be adapted for endocrine pancreatic cell induction. The filament protein nestin is expressed by neural ES cells during neural differentiation and also in a subset of immature, hormone-negative pancreatic precursors that, upon differentiation in vitro, give rise to insulin- and glucagon-expressing cells. Lumelsky et al. implemented a working protocol that began with the enrichment of the nestin-positive cell population, the population of cells derived from embryoid bodies. Embryoid bodies are structures comprising an inner layer of columnar ectoderm surrounding a proamniotic-like cavity and an outer layer of primitive endoderm. The nestin-enriched population was then expanded in serum-free medium in the presence of FGF. After withdrawal of this mitogen from the culture medium to reduce the stimulus for cell division, other factors, such as nicotinamide, previously shown to be useful in directing the differentiation and/or proliferation of precursors found in fetal pancreatic cells (47), were added. The result was the production of aggregates of cells expressing insulin.
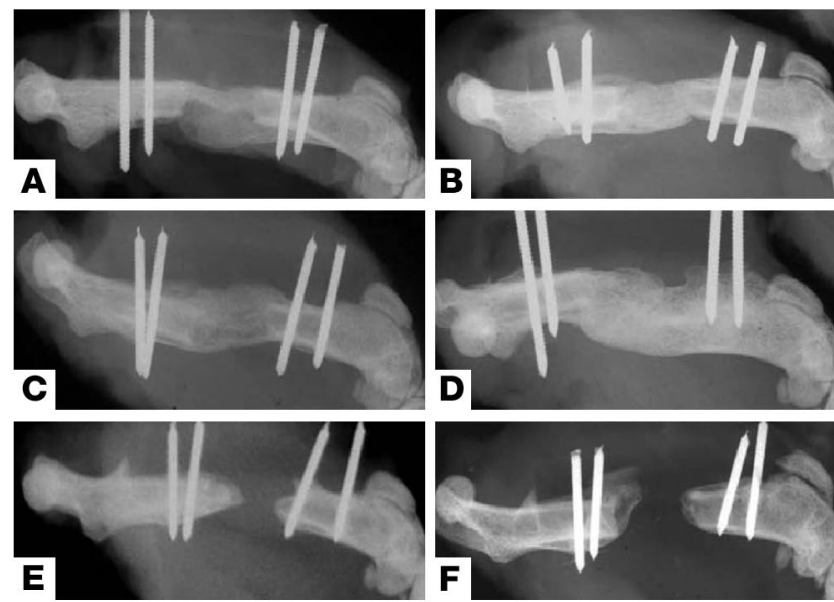

\section{Figure 5}

Radiographic evaluation at 4 and 12 weeks after surgery. The criticalsized (i.e., non-spontaneously reparable) defect in the femora treated with bone marrow-derived stromal cells transfected with retro-BMP4 exhibited a notable bridging callus (i.e., the white mass between the 2 extremes of the fracture interval) at both $4($ A) and 12 (B) weeks after surgery. The defect in the femora treated with MDCs transfected with BMP4 had also developed a bridging callus at 4 (C) and 12 (D) weeks. No bone formation was radiographically evident in the control - i.e., femora treated with MDCs transfected with the LacZ gene - at both 4 (E) and $12(\mathbf{F})$ weeks after surgery. Figure reproduced with permission from Langenbecks Archives of Surgery (55). 


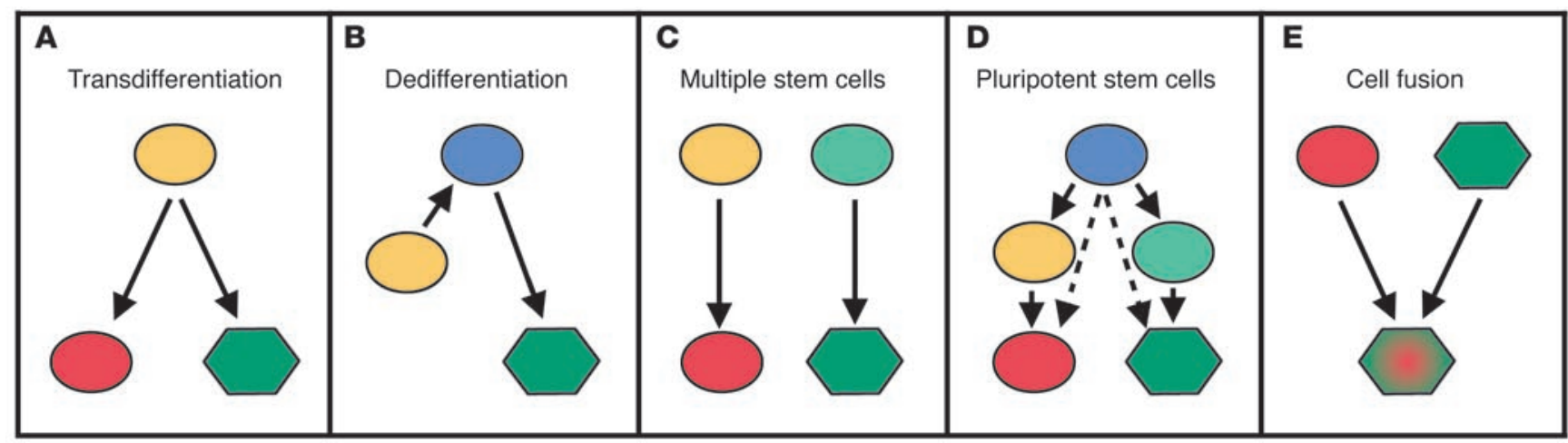

Figure 6

Schematic diagram depicting potential and known mechanisms of adult stem cell plasticity (A-E). Orange or green ovals, tissue-specific stem cells; blue ovals, pluripotent stem cells; red ovals and green hexagons, differentiated cells of tissue-specific (orange or green) lineages. Figure reproduced with permission from Cell (44).

However, in other hands, similar manipulations have failed to produce a pancreatic endocrine phenotype in nestin-positive-selected cells. Nestin-positive cells seem to contribute to the extra- and intramicrovasculature of the islet rather than to the hormone-producing cells of the pancreas themselves $(48,49)$. Further criticism of these results originated from the evidence that cells in culture may actually concentrate from the medium the insulin they seem to have produced (50). Definite answers may be obtained by repeating the same experiments using ES cells carrying transgenic fluorescent reporter genes controlled by the insulin promoter $(51,52)$.

\section{The potential of adult stem cells}

While the approach discussed above intends to recreate, using specific feeder cell layers and known growth factors, an environment similar to that existing in vivo and also to facilitate and guide a desired type of cellular differentiation, the approach in another study was to isolate in vitro adult pluripotent stem cells from any manageable source (e.g., the bone marrow) and physically introduce these cells into the already existing, appropriate environment of living recipients. By receiving spatially and temporally restricted signals from the environment, the precursors may differentiate into the cells constitutive of that specific target tissue. Lagasse et al. (53) astutely took advantage of this approach when they successfully generated hepatocytes in vivo from purified HSCs. Using a cocktail of specific antibodies able to recognize a combination of distinct cell surface markers (c-kit high Thy ${ }^{\text {low }}$ Lin $^{-}$Sca- $\left.1^{+}\right)$on a single cell, the authors physically isolated HSCs from bone marrow and transplanted them into the fumarylacetoacetate hydrolase-deficient mouse, an animal model of fatal hereditary tyrosinemia type I. Liver function in 4 out of 9 mutant mice was restored to near normal (transaminase and tyrosine levels were slightly increased), when, 3 weeks after transplantation, the standard treatment with 2-(2-nitro-4-trifluoromethylbenzyol)-1,3-cyclohexane-dione was discontinued, and these mice survived for an additional 6 months without signs of progressive liver failure or renal tubular damage. When the experiment was interrupted at 7 months after transplantation, 30-50\% of the liver mass showed cells expressing donor-derived markers (53). Similarly, by transferring precursors into the brain of a recipient mouse, Weimann et al. (54) were able to see purified HSCs differentiate into Purkinje neurons. Like HSCs, once transduced with a retrovirus to express bone morphogenetic protein 4 (BMP4), muscle-derived cell
(MDC) precursors were able to dramatically improve the healing of a spontaneously irreparable bone fracture (Figure 5) (55).

These data suggest that the signals sent via host-secreted factors or by cell-to-cell contacts are powerful enough to guide the transplanted precursors to differentiate into the same type of cells surrounding them, even across different lineages. This ability to transdifferentiate (i.e., generate a progeny belonging to a tissue lineage different from the one of origin) certainly constituted, at the time of publication, an astonishing discovery $(56,57)$. No one had previously even speculated that a mammalian stem cell, present in a mature individual, could possess such an impressive plasticity (Figure 6) (44). The possibility that new $\beta$ cells could be generated from adult stem cells isolated from tissues belonging to other lineages is particularly appealing, because it would avoid the potential ethical problems associated with the use of ES cells. However, the same authors who originally proposed transdifferentiation processes to explain their results soon realized that much of the sensational data they interpreted as results of an efficient transdifferentiation process were most likely the result of cell-to-cell fusion (Figure 6) (58-61). Others have argued that not all the results used to support the transdifferentiation capabilities of adult stem cells should be dismissed or could be solely explained by the cell fusion theory. Ianus et al. (62) deliberately entitled their article "In vivo derivation of glucose-competent pancreatic endocrine cells from bone marrow without evidence of cell fusion" (ital-

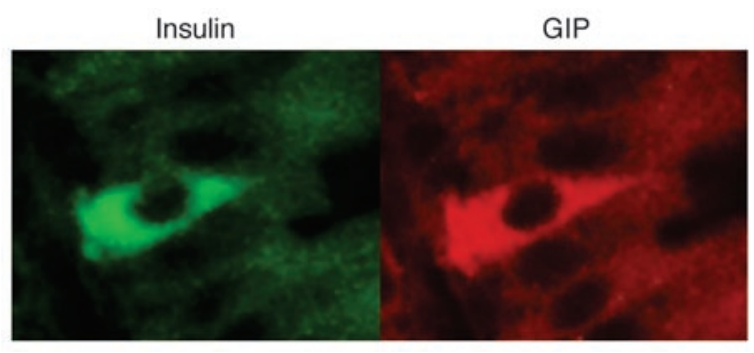

Figure 7

Duodenal sections from transgenic mice harboring the GIP/Ins transgene. The $\mathrm{K}$ cells of the gut examined by immunofluorescence microscopy showed both human insulin production (green) and expression of the glucose-dependent insulinotropic polypeptide (red). Figure reproduced with permission from Science (62). 
ics added). Regardless, it is perhaps useful to instead consider the possibility of actually forcing the fusion of transplanted precursor cells with the recipient damaged cells as a means of producing newly functional cells, even though the endocrine pancreas appears to be an environment difficult to physically reach in vivo.

\section{The potential for gene therapy}

Instead of promoting cell-to-cell fusion in an effort to restore certain physiologic functions lost with the death of specific cells, it has been considered perhaps more efficient to transfect cells belonging to a certain lineage with genes able to convert them into cells carrying the characteristics of those lost, even if they belong to a completely different lineage. In particular, in the field of diabetes research, gut K cells of the mouse were induced to produce human insulin by transfecting the human insulin gene linked to the $5^{\prime}$-regulatory region of the gene encoding glucose-dependent insulinotropic polypeptide (GIP) (Figure 7) (63). Also, research demonstrating that hepatocytes transfected with the $P d x 1$ gene under the control of the rat insulin 1 promoter were able to produce insulin $(64,65)$ attracted significant attention and has inspired new hope. In these studies, sufficient levels of insulin were secreted to satisfy the needs of a diabetic mouse, which, when treated, became and remained steadily euglycemic. These studies, however, have not yet been successfully repeated by other groups.

\section{The future problems we face}

The ability to clone human embryos and to derive from them human ES cell lines is already a reality $(41,43,45)$. Even if tomorrow's scientists could derive, by cloning, cell lines able to be differentiated toward cells with the phenotype of a $\beta$ cell and in sufficient numbers to satisfy the needs of the diabetic transplant recipient, in order that they can be used for clinical purposes, these cell lines should carry the diploid genome of somatic cell nuclei derived from individuals unrelated to the oocyte donor, i.e., those of the recipient. Further, we would still need to isolate from each patient his or her own stem cell line or develop a bank of cell lines representing the broad diversity of human $\mathrm{MHC}$ polymorphisms in order to avoid allorejection. Under these circumstances, however, the presence of autoimmunity will remain a concern. If we opt to avoid this demanding preparative effort to match donor and recipient, it would be necessary to reduce the likelihood of allorejection by other means. It may be easier to achieve this goal by using ES cells rather than any other adult cell for transplant, since the already-studied human ES cell lines appear able to downregulate the expression of MHC antigens at their surface $(66,67)$. It should still be noted, however, that rapidly dividing cells with this unique phenotype have to spontaneously stop growing once a specific, predetermined, total population cell mass has been reached, since they appear not to be controlled by NK cells (66). Furthermore, even assuming that we could overcome all of these challenges, the use of human ES cell lines tailored ad personam would constitute an extremely demanding and expensive proposition (68).

Perhaps equally demanding would be the use of means other than bone marrow transplant to evade an autoimmune response. However, some promising strategies, possibly more suitable for therapeutic applications than bone marrow transplant, have already been pro- posed. Once refined, they may allow the reestablishment of a tolerant immune state that would free the patient from the protracted attack of $\mathrm{T}$ cells against their $\beta$ cell structures and consequently from the requirement for administration of powerful immunosuppressive regimens even when transplanted with MHC-matched insulin-producing cells. While very efficient in the NOD mouse, the use of anti-CD3 monoclonal antibodies able to skew the response of diabetogenic, autoreactive T cells from a Th1 to a Th2 program (69) seems only able to slow the progression of disease to permanent diabetes in humans (70). The possible involvement of $\mathrm{CD} 4^{+} \mathrm{CD} 25^{+}$ $\mathrm{T}$ cells in the preservation of tolerance, postulated following results obtained in cured mice (71), is also the basis of the treatment proposed by Zheng and collaborators (72), which involves the administration of rapamycin, agonistic IL-2-Ig fusion protein, and a mutant, antagonist-type IL-15-related cytolytic Ig fusion protein to obtain long-term engraftment and tolerance of allogeneic islets transplanted into overtly diabetic NOD mice. The $\mathrm{CD} 4^{+} \mathrm{CD} 25^{+} \mathrm{T}$ cell population is also increased once CD40, CD80, and CD86 cell surface molecules are specifically downregulated by ex vivo treatment of NOD mice DCs with a mixture of antisense oligonucleotides targeting CD40, CD80, and CD86 primary transcripts (73). The incidence of diabetes is significantly delayed by a single injection of the engineered NOD mouse-derived DCs into syngeneic recipients. Assuming then that autoimmunity could be overcome, the potential regeneration-based treatment would still require that we determine the length of time the $\beta$ cell regenerative potential could be exploited and the characterization of possible triggering factors that would allow quantitative and chronological limits to be bypassed.

\section{Conclusion}

Our young diabetic patients must check their blood glucose levels and be injected with insulin at least 4 times a day. Concurrently, they live with the constant threat of incidents secondary to unpredictable acute hypoglycemic episodes and the ever-present worry of chronic complications. Although human ES cell research carries with it enormous scientific potential in the treatment and possible cure of many diseases, in the near future, the advances in the realm of immunoregulation may precede those in the stem cell arena. To me, finding safe ways to block autoimmunity seems to be the first goal we should achieve in order to give our patients a reliable solution to their heavy, lifelong burden, since it is a prerequisite for both the efficient use of an ES cell-based therapy and the reestablishment of euglycemia capitalizing on the pancreatic regenerative pathway.

\section{Acknowledgments}

I want to thank Len Harrison and the confidential invited peer reviewers for critically reading the paper; and Timothy Kieffer, Johnny Huard, YiGang Chang, and Tatiana Zorina for allowing me to use their photographs.

Address correspondence to: Massimo Trucco, Division of Immunogenetics, Children's Hospital of Pittsburgh, Rangos Research Center, 3460 5th Avenue, Pittsburgh, Pennsylvania 15213-3205, USA. Phone: (412) 692-6570; Fax: (412) 692-5809; E-mail: mnt@pitt.edu.
1. Wells, J.M. 2003. Genes expressed in the developing
endocrine pancreas and their importance for stem
cell and diabetes research. Diabetes Metab. Res. Rev.
19:191-201.
2. Jensen, J. 2004. Gene regulatory factors in pancreatic development. Dev. Dyn. 229:176-200.

3. Bach, J.F. 1994. Insulin-dependent diabetes mellitus as an autoimmune disease. Endocr. Rev. 15:516-542.

4. Lacy, P.E. 1982. Pancreatic transplantation as a means of insulin delivery. Diabetes Care. 5(Suppl. 1):93-97.
5. Shapiro, A.M., et al. 2000. Islet transplantation in seven patients with type 1 diabetes mellitus using a glucocorticoid-free immunosuppressive regimen. N. Eng. J. Med. 343:230-238.

6. Ryan, E.A., et al. 2001. Clinical outcomes and insulin 
secretion after islet transplantation with the Edmonton protocol. Diabetes. 50:710-719.

7. Steffensen, I., Dulin, M.F., Walters, E.T., and Morris, C.E. 1995. Peripheral regeneration and central sprouting of sensory neurone axons in Aplysia californica following nerve injury. J. Exp. Biol. 198:2067-2078.

8. Ramachandra, A.V., Swamy, M.S., and Kurup, A.K 1996. Local and systemic alterations in cyclic 3',5 AMP phosphodiesterase activity in relation to tail regeneration under hypothyroidism and $\mathrm{T} 4$ replacement in the lizard, Mabuya carinata. Mol. Reprod. Dev. 45:48-51.

9. Stocum, D.L. 2002. A tail of transdifferentiation. Science. 298:1901-1903.

10. Lipsett, M., and Finegood, D.T. 2002. $\beta$-cell neogenesis during prolonged hyperglycemia in rats. Diabetes. 51:1834-1841.

11. Gu, D., Lee, M.S., Krahl, T., and Sarvetnick, N. 1994. Transitional cells in the regenerating pancreas. Devel opment. 120:1873-1881.

12. Bernard-Kargar, C., and Ktorza, A. 2001. Endocrine pancreas plasticity under physiological and pathological conditions. Diabetes. 50 (Suppl. 1):S30-S35.

13. Brelje, T.C., et al. 1993. Effect of homologous placental lactogens, prolactins, and growth hormones on islet $\beta$-cell division and insulin secretion in rat, mouse, and human islets: implication for placental lactogen regulation of islet function during pregnancy. Endocrinology. 132:879-887.

14. Rosenberg, L. 1995. In vivo cell transformation: neogenesis of beta cells from pancreatic ductal cells. Cell Transplant. 4:371-383.

15. Bouwens, L. 1998. Transdifferentiation versus stem cell hypothesis for the regeneration of islet beta-cells in the pancreas. Microsc. Res. Tech. 15:332-336.

16. Bonner-Weir, S., Deery, D., Leahy, J.L., and Weir, G.C. 1989. Compensatory growth of pancreatic betacells in adult rats after short-term glucose infusion. Diabetes. 38:49-53.

17. Arnush, M., et al. 1996. Growth factors in the regenerating pancreas of gamma-interferon transgenic mice. Lab. Invest. 74:985-990.

18. Herrera, P.L. 2000. Adult insulin- and glucagon-producing cells differentiate from two independent cell lineages. Development. 127:2317-2322.

19. Gu, G., Dubauskaite, J., and Melton, D.A. 2002. Direct evidence for the pancreatic lineage: $\mathrm{NGN}^{+}$cells are islet progenitors and are distinct from duct progenitors. Development. 129:2447-2457.

20. Weir, G.C., and Bonner-Weir, S. 2004. Beta-cell precursors - a work in progress. Nat. Biotechnol. 22:1-2.

21. Seaberg, R.M., et al. 2004. Clonal identification of multipotent precursors from adult mouse pancreas that generate neural and pancreatic lineages. Nat. Biotechnol. 22:1115-1124.

22. Suzuki, A., Nakauchi, H., and Taniguchi, H. 2004. Prospective isolation of multipotent pancreatic progenitors using flow-cytometric cell sorting. Diabetes. 53:2143-2152.

23. Bonner-Wier, S., and Sharma, A. 2002. Pancreatic stem cells. J. Pathol. 197:519-526.

24. Dor, Y., Brown, J., Matinez, O.I., and Melton, D. 2004. Adult pancreatic beta-cells are formed by self-duplication rather than stem-cell differentiation. Nature. 429:41-46.

25. Pelengaris, S., Khan, M., and Evan, G.I. 2002. Suppression of Myc-induced apoptosis in $\beta$ cells exposes multiple oncogenic properties of Myc and triggers carcinogenic progression. Cell. 109:321-334.

26. Sutherland, D.E., et al. 1984. Twin-to-twin pancreas transplantation: reversal and reenactment of the pathogenesis of type I diabetes. Trans. Assoc. Am. Physi cians. 97:80-87.

27. Ogawa, N., List, J.F., Habener, J.F., and Maki, T. 2004. Cure of overt diabetes in NOD mice by transient treatment with anti-lymphocyte serum and exendin-4. Diabetes. 53:1700-1705.
28. Ikehara, S., et al. 1985. Prevention of type 1 diabetes in nonobese diabetic mice by allogeneic bone marrow transplantation. Proc. Natl. Acad. Sci. U. S. A. 82:7743-7747.

29. Li, H., et al. 1996. Mixed allogeneic chimerism induced by a sublethal approach prevents autoimmune diabetes and reverses insulitis in nonobese diabetic (NOD) mice. J. Immunol. 156:380-388.

30. Zorina, T.D., et al. 2002. Distinct characteristics and features of allogeneic chimerism in the NOD mouse model of autoimmune diabetes. Cell Transplant. 11:113-123.

31. Ryu, S., Kodama, S., Ryu, K., Schoenfeld, D.A., and Faustman, D.L. 2001. Reversal of established autoimmune diabetes by restoration of endogenous $\beta$ cell function. J. Clin. Invest. 108:63-72. doi:10.1172/ JCI200112335.

32. Hess, D., et al. 2003. Bone marrow-derived stem cells initiate pancreatic regeneration. Nat. Biotechnol. 21:763-770.

33. Zorina, T.D., et al. 2003. Recovery of the endogenous beta cell function in autoimmune diabetes. Stem Cells. 21:377-388.

34. Ricordi, C., et al. 1991. In vivo effect of FK506 on human pancreatic islets. Transplantation. 52:519-526.

35. Tamura, K., et al. 1995. Transcriptional inhibition of insulin by FK506 and possible involvement of FK506 binding protein- 12 in pancreatic $\beta$-cell. Transplantation. 59:1606-1615.

36. Bell, E., et al. 2003. Rapamycin has a deleterious effect on MIN-6 cells and rat and human islets. Diabetes. 52:2731-2739.

37. Paris, M., Bernard-Kargar, C., Berthault, M.F., Bouwens, L., and Ktorza, A. 2003. Specific and combined effects of insulin and glucose on functional pancreatic $\beta$-cell mass in vivo and in adult rats. Endocrinology. 144:2717-2727.

38. Farilla, L., et al. 2002. Glucagon-like peptide-1 promotes islet cell growth and inhibits apoptosis in Zucker diabetic rats. Endocrinology. 143:4397-4408.

39. Lechner, A., et al. 2004. No evidence for significant transdifferentiation of bonemarrow into pancreatic $\beta$-cells in vivo. Diabetes. 53:616-623.

40. Kodama, S., Kühtreiber, W., Fujimura, S., Dale, E.A., and Faustman, D.L. 2003. Islet regeneration during the reversal of autoimmune diabetes in NOD mice. Science. 302:1223-1227.

41. Thomson, J.A., et al. 1998. Embryonic stem cell lines derived from human blastocysts. Science. 282:1145-1147.

42. Shamblott, M.J., et al. 1998. Derivation of pluripotent stem cells from cultured human primordial germ cells. Proc. Natl. Acad. Sci. U. S. A. 95:13726-13731.

43. Cowan, C.A., et al. 2004. Derivation of embryonic stem-cell lines from human blastocysts. N. Engl. J. Med. 350:1353-1356.

44. Wagers, A.J., and Weissman, I.L. 2004. Plasticity of adult stem cells. Cell. 116:639-648.

45. Hwang, W.S., et al. 2004. Evidence of a pluripotent human embryonic stem cell line derived from a cloned blastocyst. Science. 303:1669-1674.

46. Lumelsky, N., et al. 2001. Differentiation of embryonic stem cells to insulin-secreting structures similar to pancreatic islets. Science. 292:1389-1394.

47. Beattie, G.M., Rubin, J.S., Mally, M.I., Otonkoski, T., and Hayek, A. 1996. Regulation of proliferation and differentiation of human fetal pancreatic islet cells by extracellular matrix, hepatocyte growth factor, and cell-cell contact. Diabetes. 45:1223-1228.

48. Selander, L., and Edlund, H. 2002. Nestin is expressed in mesenchymal and not epithelial cells of the developing mouse pancreas. Mech. Dev. 113:189-192.

49. Treutelaar, M.K., et al. 2003. Nestin-lineage cells contribute to the microvasculature but not endocrine cells of the islet. Diabetes. 52:2503-2512.

50. Rajagopal, J., Anderson, W.J., Kume, S., Martinez, O.I., and Melton, D.A. 2003. Insulin staining of ES cell progeny from insulin uptake. Science. 299:363.

51. Hara, M., et al. 2003. Transgenic mice with green fluorescent protein-labeled pancreatic beta -cells. Am. J. Physiol. Endocrinol. Metab. 284:E177-E183.

52. Bertera, S., et al. 2003. Body window-enabled in vivo multicolor imaging of transplanted mouse islets expressing an insulin-timer fusion protein. BioTechniques. 35:718-722.

53. Lagasse, E., et al. 2000. Purified hematopoietic stem cells can differentiate into hepatocytes in vivo. Nat. Med. 6:1229-1234

54. Weimann,J.M., Charlton, C.A., Brazelton, T.R., Hackman, R.C., and Blau, H.M. 2003. Contribution of transplanted bone marrow cells to Purkinje neurons in human adult brains. Proc. Natl. Acad. Sci. U. S. A. 100:2088-2093.

55. Rose, T., et al. 2003. The role of cell type in bone healing mediated by ex vivo gene therapy. Langenbecks Arch. Surg. 388:347-355.

56. Kahn, A. 2000. Converting hepatocytes to $\beta$-cells - a new approach for diabetes? (News $\&$ Views). Nat. Med. 6:505-506.

57. Perry, D. 2000. Patients' voices: the powerful sound in stem cell debate. Science. 287:1423.

58. Wagers, A.J., Sherwood, R.L., Christensen, J.L., and Weissman, I.L. 2002. Little evidence for developmental plasticity of adult hematopoietic stem cells. Science. 297:2256-2259.

59. Wang, X., et al. 2003. Cell fusion is the principal source of bone-marrow-derived hepatocytes. Nature. 422:897-901.

60. Choi, J.B., et al. 2003. Little evidence of transdifferentiation of bone marrow-derived cells into pancreatic beta cells. Diabetologia. 46:1366-1374.

61. Alvarez-Dolado, M., et al. 2003. Fusion of bone-marrow-derived cells with Purkinje neurons, cardiomyocytes and hepatocytes. Nature. 425:968-973.

62. Ianus, A., Holz, G.G., Theise, N.D., and Hussain, M.A. 2003. In vivo derivation of glucose-competent pancreatic endocrine cells from bone marrow without evidence of cell fusion. J. Clin. Invest. 111:843-850. doi:10.1172/JCI200316502.

63. Cheung, A.T., et al. 2000. Glucose dependent insulin release from genetically engineered K cells. Science. 290:1959-1962.

64. Ferber, S., et al. 2000. Pancreatic and duodenal homeobox gene 1 induces expression of insulin genes in liver and ameliorates streptozotocin-induced hyperglycemia. Nat. Med. 6:568-572.

65. Ber, I., et al. 2003. Functional, persistent, and extended liver to pancreas transdifferentiation. J. Biol. Chem. 278:31950-31957.

66. Drukker, M., et al. 2002. Characterization of the expression of MHC proteins in human embryonic stem cells. Proc. Natl. Acad. Sci. U. S. A. 99:9864-9869.

67. Li, L., et al. 2004. Human embryonic stem cells possess immune-privileged properties. Stem Cells. 22:448-456.

68. Kennedy, D. 2004. Stem cells, redux. Science. 303:1581.

69. Chatenoud, L., Primo, J., and Bach, J.-F. 1997. CD3 antibody-induced dominant self tolerance in overt autoimmunity in non-obese diabetic mice. J. Immunol. 158:2947-2954

70. Herold, K.C., et al. 2002. Anti-CD3 monoclonal antibody in new-onset type 1 diabetes mellitus. N. Engl. J. Med. 346:1692-1698.

71. Chatenoud, L. 2003. CD3-specific antibody-induced active tolerance. Nat. Rev. Immunol. 3:123-132.

72. Zheng, X.X., et al. 2003. Favorably tipping the balance between cytopathic and regulatory $\mathrm{T}$ cells to create transplantation tolerance. Immunity. 19:503-514.

73. Machen, J., et al. 2004. Antisense oligonucleotide downregulating co-stimulation confer diabetes-preventive properties to NOD dendritic cells. J. Immunol. 173:4331-4341. 